\title{
Hongos Productores de Micotoxinas Asociados a Hierbas Medicinales en la Ciudad de São Paulo, Brasil
}

\author{
(Mould and Mycotoxins Associated in Medicinal Plants in São Paulo, \\ Brazil)
}

\author{
Fabiane C. Nunes ${ }^{1}$, Patricio Godoy-Martínez ${ }^{*}$, Milton Urrutia ${ }^{3}$, \\ Benedito Correa $^{4} \&$ Olga Fischman Gompertz ${ }^{1 *}$
}

1-Disciplina de Biología Celular, Universidade Federal de São Paulo, São Paulo, Brasil 2- Instituto de Microbiología Clínica, Universidad Austral de Chile - Valdivia, Chile 3-Universidad de Antofagasta 4- Departamento de Microbiología, Universidade de São Paulo, São Paulo, Brasil. *Autor para correspondencia: patricio.godoy@uach.cl/olga.fischman@ecb.epm.br

RECIBIDO:03 de Noviembre de 2016 APROBADO: 16 de Noviembre de 2016

DOI:10.22370/bolmicol.2016.31.2.486

LOS AUTORES DECLARAN NO TENER CONFLICTO DE INTERESES

Palabras clave: Aflatoxinas; Aspergillus flavus; Aspergillus parasiticus; Fitoterápicos; Micotoxinas. Key words: Aflatoxins; Aspergillus flavus; Aspergillus parasiticus; Phytotherapy; Mycotoxins.

\section{RESUMEN}

Antecedentes: La fitoterapia es una de las más antiguas prácticas utilizadas por la humanidad. Hasta mediados del siglo XIX, cuando se introdujeron los medicamentos, la formulación de estos generalmente era basada en plantas medicinales.

Objetivos: Determinar la micobiota y los niveles de aflatoxinas originadas de Aspergillus sección Flavi aislados de las 50 muestras de medicamentos fitoterápicos comercializados actualmente en la ciudad de São Paulo, Brasil.

Métodos: Cincuenta (50) muestras de medicamentos fitoterápicos en la forma de hojas (té-25) y cápsulas (25) fueron colectadas de agosto de 2000 a julio de 2001. Los hongos filamentosos aislados fueron identificados al nivel de género de acuerdo con las características morfológicas y criterios taxonómicos. El análisis de aflatoxinas fue realizada por cromatografía de capa fina (TLC). Resultados: El análisis microbiológico mostró que 41 (82\%) de los medicamentos fitoterápicos presentaron un crecimiento fúngico sobre las $100 \mathrm{UFC} / \mathrm{g}$. Un total de 106 especies de seis diferentes géneros fueron aislados (Aspergillus, Penicillium, Mucor, Rhizopus y Alternaria). El género Aspergillus fue el predominante $(60.5 \%)$ seguido por Penicillium (20,0\%). Aspergillus niger (30\%) A. flavus (22\%), A. fumigatus $(6,5 \%)$ y $A$. parasiticus fueron las especies de Aspergillus identificadas. Se observó que $13(56,5 \%)$, de los $23 \mathrm{~A}$. flavus aislados y dos aislados de $A$. parasiticus produjeron aflatoxinas.

Conclusiones: La contaminación observada en la 
mayoría de los productos y el alto nivel de cepas productoras de aflatoxinas justifica un análisis más cuidadoso de los medicamentos fitoterápicos comercializados y la aplicación de leyes más rigurosas son necesarias para garantizar la calidad de los productos.

\section{ABSTRACT}

Background: Phytotherapy is one of the most ancient practices used by humanity. In Antiquity until the middle of the XIX century, when chemotherapeutic drugs were introduced, formulation of medicines was usually based on medicinal plants.

Objective: To determine mycobiota and levels of Aspergillus section Flavi aflatoxins isolated from 50 samples of phytotherapeutic remedies currently commercialized in São Paulo, Brazil.

Methods: Fifty (50) samples of phytotherapeutic remedies in the form of leaves (teas-25) and powders (capsules-25) were collected from August 2000 to July 2001. Filamentous fungi isolates were identified at the genera level in accordance with morphological characteristics and taxonomic criteria. Aflatoxins were performed by Thin-layer chromatography (TLC). Results: The microbiological analysis showed that $41(82 \%)$ of phytotherapeutic remedies presented a fungal growth over $100 \mathrm{CFU} / \mathrm{g}$. A total of 106 species of six different genera were isolated (Aspergillus, Penicillium, Mucor, Rhizopus and Alternaria). The genus Aspergillus was the predominant $(60.5 \%)$ followed by Penicillium genus (20.0\%). Aspergillus niger (30\%) A. flavus (22\%), A. fumigatus (6.5\%) and A. parasiticus were the species of Aspergillus identified. It was observed that $13(56.5 \%)$ of $23 \mathrm{~A}$. $\mathrm{fla}$ $v u s$ isolates and two $A$. parasiticus isolates produced aflatoxins.

Conclusions: The contamination observed in most products and the high level of aflatoxigenic strains justify the concern regarding the execution of more careful analyzes of the commercialized phytotherapeutic remedies and the application of more rigorous laws that may warrant the quality of these products.

\section{INTRODUCCIÓN}

Hasta mediados del siglo XIX las drogas y sus fórmulas farmacéuticas estaban constituidas principalmente por plantas medicinales ${ }^{1}$. A fines del siglo XX, las plantas volvieron a ocupar un importante lugar en la medicina, utilizadas especialmente por vía oral en formatos de cápsulas, extractos líquidos y en forma de infusión (té), esta última es la presentación más difundida en el mundo, por ser de fácil acceso a ella y por su simple preparación ${ }^{2,3}$. La Organización Mundial de la Salud (OMS) estima que sobre el $80 \%$ de la población mundial utiliza más de 25.000 especies de plantas en la preparación de medicamentos tradicionales ${ }^{4}$. En muchos países, las hierbas medicinales pueden ser registradas como suplementos alimenticios para dietas y pueden ser comercializadas exentas de licencias ${ }^{5}$.

Se han descrito efectos adversos por la utilización de hierbas medicinales al ser adulteradas con componentes tóxicos o contaminados por patógenos microbianos o micotoxinas que han sido descritas en productos naturales como hierbas y plantas $6,7,8,9,10,11$. Muy poca información existe sobre la micoflora y micotoxinas aisladas de medicamentos fitoterápicos y su asociación con problemas de calidad. El presente estudio determinó los hongos aislados en 50 medicamentos fitoterápicos normalmente comercializados en la ciudad de São Paulo, Brasil y los niveles de micotoxinas producidos por estos.

\section{MATERIAL Y MÉTODOS}

\section{Muestras}

Cincuenta (50) muestras de fitoterápicos en la forma de hojas (té-25) y cápsulas (25) fueron obtenidas de farmacias de manipulación autorizadas, 
vendedores callejeros y distribuidores de materia prima en la ciudad de São Paulo (Brasil).

Cinco diferentes hierbas con distintas acciones y propiedades farmacológicas fueron analizadas: Cynara scolymus, Peumus boldus, Maytenus ilicifolia, Cassia acutifolia y Rhamnus purshiana.

\section{Aislamiento del hongo}

La cuantificación de los propágulos fúngicos fue efectuado en medio sólido utilizando el método de cuantificación en superficie. Diez (10) gramos de cada muestra fueron mecánicamente homogenizados en $90 \mathrm{~mL}$ de agua peptonada con buffer (MERCK) por 30 minutos. Diluciones seriadas $10^{-2}$ hasta $10^{-6}$ fueron alicuotadas en $0.1 \mathrm{~mL}$ depositadas sobre la superficie de agar Sabouraud dextrosa (DIFCO) con cloranfenicol por triplicado. Las placas fueron incubadas invertidas a $26^{\circ} \mathrm{C} \pm$ $1^{\circ} \mathrm{C}$ por 7 días. Después de la incubación, las colonias fueron contadas, y las unidades formadoras de colonias (UFC) por gramo fueron calculadas ${ }^{12,13}$.

\section{Identificación}

Los hongos filamentosos aislados fueron identificados al nivel de género de acuerdo con las características morfológicas y criterios taxonó$\operatorname{micos}^{14,15}$. Todos los aislados de Aspergillus fueron examinados en agar extracto de malta (MEA) y agar Czapeck (CYA). Aspergillus de la sección Flavi (A. flavus y A. parasiticus) fueron identificados de acuerdo a las claves de Klich y Pitt16.

\section{Ensayo para la detección de micotoxinas de los hongos aislados}

Aspergillus de la sección Flavi y especies del género Penicillium fueron analizados para verificar la capacidad de producir micotoxinas (aflatoxinas) utilizando agar coco (CAM)17. Cada "plug" de micelio de la colonia fue transferida para una placa de CAM y después incubada en oscuridad a $28^{\circ} \mathrm{C}$. Las placas fueron examinadas al cuarto y décimo día, bajo luz ultravioleta (UV) a $365 \mathrm{~nm}$ (UVSL 25-Mineral Light) para observar la presen- cia de fluorescencia característica. El crecimiento fúngico en CAM de todos los aislados fueron triturados con $30 \mathrm{~mL}$ de cloroformo y la mezcla fue filtrada con papel Whatman $\mathrm{N}^{0} 1$ y concentrada hasta el volumen de $3 \mathrm{~mL}$, al vacío, en un evaporador a $60^{\circ} \mathrm{C}$.

\section{Análisis de aflatoxinas}

El análisis de micotoxinas fue realizada por cromatografía de capa fina (TLC), siguiendo la metodología propuesta por Soares y RodriguezAmaya $^{18}$. El extracto obtenido de los cultivos de CAM se analizaron por TLC, un volumen de $5 \mathrm{uL}$ y depositados sobre una placa de sílica gel 60G, 20 x $20 \mathrm{~cm}$ (Merck, Darmstadt, Germany) con 0.25$\mathrm{mm}$ de grosor $^{19}$. Las aflatoxinas B1, B2, G1 y G2 (Sigma Chemical Co., St. Louis, Missouri, USA) fueron utilizadas como estándar. Las placas fueron reveladas con tolueno: etil acetato: cloroformo: 90\%, ácido fórmico (70:50:50:20, v/v)20. Las aflatoxinas se detectaron bajo luz UV (365 nm). Se realizó la comparación de los valores (Rf) de los extractos con los estándares. La confirmación de las aflatoxinas fue llevada a cabo por la reacción de ácido trifluoroacético ${ }^{21}$.

\section{Análisis Estadístico}

El programa estadístico R (versión 2.1.1, R DEVELOPMENT CORE TEAM, 2005) fue utilizado para analizar los datos obtenidos en el presente estudio. La concentración de las aflatoxinas producidas por las especies de A. flavus son presentadas como media y desviación estándar. Lo que concierne a $A$. parasiticus son presentadas como media e intervalo intercuartil. Para verificar si existen diferencias significativas entre muestras inadecuadas siendo utilizada como hipótesis nula en una proporción de 50\%. El test t de Student's fue utilizado para determinar las diferencias significativas entre la concentración de aflatoxinas para A. flavus y el límite establecido por el Ministerio de Agricultura y Abastecimiento del Brasil ${ }^{22}$. Para $A$. parasiticus, la concentración de aflatoxinas sigue 
los mismos principios informados anteriormente; las pruebas de Mann-Whitney y Fisher's exacto fue utilizada para determinar el grado de asociación entre contaminación y la forma del consumo del té. La significancia estadística fue de $5 \%$.

\section{RESULTADOS}

En el análisis de las 50 muestras de medicamentos fitoterápicos, en forma de hojas (té) y cápsulas usadas en la ciudad de São Paulo, éste mostró la presencia de 106 agentes fúngicos. Aspergillus fue el principal género aislado $(60,5 \%)$, siendo sus especies identificadas como $A$. niger (30\%), A. flavus (22\%), A. fumigatus (6,5\%) y A. parasiticus (2\%), otros géneros identificados fueron Penicillium spp. (20\%), Rhizopus spp. (6.5\%) y Alternaria (4\%) (Tabla I).

El índice de contaminación de las hojas y cápsulas fue similar; por esta razón el análisis de los productos es presentado en conjunto. El número de colonias fúngicas varió entre 1x102 a $5 \times 104 \mathrm{UFC} / \mathrm{g}$ siendo la proporción de muestras inadecuadas significativamente mayor que la de las muestras adecuadas. En nuestra investigación el $82 \%$ de las muestras presentaron crecimiento fúngico sobre $100 \mathrm{UFC/g}$, lo que indica una diferencia estadística significativa respecto al de la hipótesis nula de $50 \%$ con $\mathrm{P} \leq 0.0001$ (Tabla II).
De las 10 muestras de materia prima analizadas, 5 habían sido esterilizadas previamente y presentaron condiciones apropiadas para el consumo.

De las 30 muestras vendidas en las farmacias, apropiadas para el consumo, solamente 4 $(13,33 \%)$ tenían condiciones para el consumo.

De las 10 muestras (fitoterápicos) obtenidas de los vendedores callejeros (ambulantes) el $100 \%$ de estas mostraron niveles inadecuados para el consumo (Tabla II).

De las 23 cepas de A. flavus, 13 (56,5\%) produjeron aflatoxinas: $10(77 \%)$ produjeron simultáneamente aflatoxina B1 (AFB1) y aflatoxina B2 (AFB2), cuyos niveles variaron entre 4,6 a $19,7 \mu \mathrm{g} / \mathrm{kg}$ para AFB1 y entre 1,5 a $8,0 \mu \mathrm{g} / \mathrm{kg}$ para AFB2. Tres aislados de A. flavus expresaron solamente aflatoxina B1 en niveles de 7,0 a 16,8 $\mu \mathrm{g}$ / $\mathrm{kg}$, (Tabla III). La producción de AFB1 fue significativamente mayor que el máximo nivel de contaminación e indica diferencias estadísticamente significativas respecto del valor máximo de $5 \mu \mathrm{g} /$ $\mathrm{kg}$, para $\mathrm{P} \leq 0.0001$. Las dos cepas de $A$. parasiticus expresaron aflatoxinas AFB1, AFB2, AFG1, AFG2 con una concentración entre 14,1 y $15,0 \mu /$ $\mathrm{kg}$ para AFB1; 1,2 y $1,5 \mu \mathrm{g} / \mathrm{kg}$ para $\mathrm{AFB} 2 ; 5,0 \mathrm{y}$ $7,0 \mu \mathrm{g} / \mathrm{kg}$ para AFG1, 2,2 y 3,2 $\mu \mathrm{g} / \mathrm{kg}$ para AFG2 (Tabla IV).

Tabla 1. Distribución de los agentes fúngicos aislados de medicamentos fitoterápicos.

\begin{tabular}{lcc}
\hline Géneros & $\mathrm{N}$ & $\%$ \\
\hline Aspergillus spp. & 64 & 60,5 \\
Penicillium spp. & 21 & 20,0 \\
Mucor spp. & 10 & 9,0 \\
Rhizopus spp. & 7 & 6,5 \\
Alternaria spp. & 4 & 4,0 \\
\hline Total & 106 & 100 \\
\hline
\end{tabular}


Tabla 2. Distribución de las muestras de los medicamentos fitoterápicos de acuerdo a su clasificación para el consumo y local de comercialización.

\begin{tabular}{|c|c|c|c|c|c|c|c|c|}
\hline \multirow{3}{*}{$\begin{array}{c}\text { Clasificación } \\
\text { UFC/g }\end{array}$} & \multicolumn{6}{|c|}{ Sitio de Venta } & \multirow{2}{*}{\multicolumn{2}{|c|}{ Total }} \\
\hline & \multicolumn{2}{|c|}{ Calle } & \multicolumn{2}{|c|}{ Farmacia } & \multicolumn{2}{|c|}{ Distribuidor } & & \\
\hline & $\mathrm{N}$ & $\%$ & $\mathrm{~N}$ & $\%$ & $\mathrm{~N}$ & $\%$ & $\mathrm{~N}$ & $\%$ \\
\hline Adecuada $<10^{2}$ & 0 & 0,00 & 4 & 13,33 & $5^{\&}$ & 50,00 & 9 & 18,00 \\
\hline Inadecuada $>10^{2}$ & 10 & 100,00 & 26 & 86,67 & 5 & 50,00 & 41 & $82,00 *$ \\
\hline Total & 10 & 100,00 & 30 & 100,00 & 10 & 100,00 & 50 & 100,00 \\
\hline
\end{tabular}

* Indica diferencias estadisticamente significativas, en relación a hipótesis nula en una distribución igual a $50 \%$ para $\mathrm{P} \leq 0.0001$.

$\mathrm{N}=$ número de muestras analizadas

$\&=$ muestras previamente esterilizadas

Tabla 3. Concentración de toxinas de las muestras de A. flavus aisladas de los medicamentos fitoterápicos

\begin{tabular}{|c|c|c|c|}
\hline \multirow{2}{*}{ Aislado } & \multicolumn{2}{|c|}{ Media $(\mu \mathrm{g} / \mathrm{kg})$} & \multirow{2}{*}{$\begin{array}{c}\text { Total }(\mu \mathrm{g} / \mathrm{kg}) \\
(\mathrm{AFB} 1+\mathrm{AFB} 2)\end{array}$} \\
\hline & AFB1 & $\mathrm{AFB} 2$ & \\
\hline 1 & 19,7 & 8,0 & 27,7 \\
\hline 2 & 16 & 5,0 & 21,0 \\
\hline 3 & 10,5 & 1,6 & 12,1 \\
\hline 4 & 14,8 & 1,5 & 16,3 \\
\hline 5 & 17,5 & 3,11 & 20,6 \\
\hline 6 & 13,3 & 7,5 & 20,8 \\
\hline 7 & 4,6 & 1,5 & 6,1 \\
\hline 8 & 15,7 & 4,8 & 20,5 \\
\hline 9 & 14,5 & 2,8 & 17,3 \\
\hline 10 & 12,5 & 1,7 & 14,2 \\
\hline 11 & 13,6 & ND & 13,6 \\
\hline 12 & 16,8 & ND & 16,8 \\
\hline 13 & 7,0 & ND & 7,0 \\
\hline Descriptivo & $13,58(4,19) *$ & $3,75(2,47)$ & $16,46(5,98)$ \\
\hline
\end{tabular}

Bol. Micol. 2016; 31(2): 1-8

micologia.uv.cl 
Tabla 4. Concentración de toxinas de aislados de A. parasiticus obtenidos de medicamentos fitoterápicos

\begin{tabular}{lccccc}
\hline \multirow{2}{*}{ Aislado } & \multicolumn{4}{c}{ Media $(\mu \mathrm{g} / \mathrm{kg})$} & Total $(\mu \mathrm{g} / \mathrm{kg})$ \\
\cline { 2 - 5 } & AFB1 & AFB2 & AFG1 & AFG2 & (AFB1 + AFB2 + AFG1 + AFG2) \\
\hline 14 & 14,1 & 1,2 & 7,0 & 3,2 & 25,5 \\
15 & 15,0 & 1,5 & 5,0 & 2,2 & 23,7 \\
\multirow{2}{*}{ Media } & 14,5 & 1,4 & 6,0 & 2,7 & 24,6 \\
\hline
\end{tabular}

Límite para $\mathrm{AFB} 1=5 \mu \mathrm{g} / \mathrm{kg}$. Límite para $\mathrm{AFB} 1+\mathrm{AFB} 2=20 \mu \mathrm{g} / \mathrm{kg}$.

\section{DISCUSIÓN}

El control de calidad microbiológico de los alimentos determina que un producto adecuado para el consumo debe contener un máximo de 100 UFC del hongo/g ${ }^{23}$. En nuestra investigación, el $82 \%$ de las muestras en forma de hojas y cápsulas presentaron similares índices de contaminación, estando sobre el límite establecido, siendo inadecuado para el consumo humano. Analizando la micoflora de 50 muestras de medicamentos fitoterápicos fueron aislados un total de 106 hongos, con una prevalencia de los géneros Aspergillus y Penicillium. En Brasil, índices de toxinas han sido analizados especialmente en maíz. Corrêa et al. ${ }^{24}$ encontraron contaminación por aflatoxinas en $56 \%$ de un total 600 muestras de maíz en el estado de Paraná, Goiás y Mato Grosso do Sul. Amorim et al. ${ }^{25}$ observaron la presencia de aflatoxinas en $98 \%$ de un total de 264 muestras de maíz obtenidas de varios estados Brasileros.

De los 23 aislados de $A$. flavus en el presente estudio, $13(56,5 \%)$ fueron productores de aflatoxinas. En la literatura disponible encontramos escasas referencias sobre contaminación de medicamentos fitoterápicos utilizados en la forma de hojas (té). Aislamos 2 muestras de $A$. parasiticus que junto con $A$. flavus son los principales agentes productores de aflatoxinas. Analizando 48 muestras de té in Omán (Persia), Abdulkadir et al. ${ }^{26}$ identificaron 25 cultivos de $A$. flavus los cua- les no mostraron toxicidad. Tassaneeyakul et al. ${ }^{27}$ verificaron la presencia de aflatoxinas en $18 \%$ de hierbas medicinales en Tailandia. Esta frecuencia es similar a lo observado por Halt en estudio realizado en Croacia (1998) donde analizó 62 muestras de hierbas medicinales ${ }^{28}$. Sin embargo, en nuestros resultados $56,5 \%$ de estos productos presentaron contaminación por aflatoxinas.

En el presente estudio AFB1 fue la toxina más detectada con elevada concentración. De los 13 aislados de $A$. flavus solamente una presentó un nivel inferior a $5 \mu \mathrm{g} / \mathrm{kg}$ de AFB1. Detectamos 4 aflatoxinas (AFB1, AFB2, AFG1, AFG2) en 2 aislados de $A$. parasiticus con niveles de AFB1 mayores a los establecidos por la Food and Agriculture Organization of the United Nations $(\mathrm{FAO})^{29}$. El pequeño número de aislados no permitió una evaluación estadística. En nuestro estudio, Penicillium spp. fue aislado en $20 \%$ de todas las muestras analizadas, no fueron aisladas cepas productoras de micotoxinas. Altos índices de contaminación por Penicillium spp, han sido encontrados en cereales, maíz y granos, todos componentes de la dieta humana ${ }^{30,31}$.

Es importante enfatizar que la presencia de hongos toxigénicos en hierbas medicinales y derivados puede representar un factor de riesgo importante para la población que los consume regularmente, principalmente en niños, ancianos y pacientes inmunodeprimidos. La contaminación por hongos fue observada en la mayoría de los 
productos analizados $(82 \%)$ y la producción de micotoxinas por parte de estos, justifica la intensificación del control de calidad para los medicamentos fitoterápicos ampliamente utilizados en la medicina alternativa y la aplicación de leyes más rigurosas que permitan garantizar calidad en orden de entregar al consumidor productos apropiados para el consumo ${ }^{32,33}$.

\section{AGRADECIMIENTOS}

Este estudio fue financiado por la Coordenação de Aperfeiçoamento de Pessoal de Ensino Superior (CAPES) y Fundação de Amparo à Pesquisa do Estado de São Paulo (FAPESP Proceso $n^{\circ}$ 00/14084-1).

\section{REFERENCIAS}

1. Guyot, MM. (1990). Perspectivas de la fitoterapia. Acta Farm Bon. 9(2): 131-138.

2. Araújo, ALA., Ohara, MT. (2000). Qualidade microbiológica de drogas vegetais comercializadas em feira de São Paulo e de infusos derivados. Rev Bras Cienc Farm. 36: 129-137.

3. Brandão, MGL., Oliveira, P., Moreira, RA., et al. (2001). Qualidade de amostras comerciais e produtos fitoterápicos: Drogas inscritas na farmacopéia brasileira. Infarma. 13: 60-61.

4. World Health Organization. (1992). Quality control methods for medicinal plants materials. Geneva, WHO: 122.

5. Barnes, J. (2003). Quality, efficacy and safety of complementary medicines: fashions, facts and the future. Part I. Regulation and quality. Br J Clin Pharmacol. 55: 226-233.

6. Boullata, J I., Nace, A M. (2000). Safety issues with herbal medicine. Pharmacotherapy. 20: 257-269.

7. Elvin-Lewis, M. (2001). Should we be concerned about herbal remedies. J Ethnopharmacol. 75 (2-3): 141-164.

8. Kneifel, W., Czech, E., Kopp, B. (2002). Microbial contamination of medicinal plants - a review. Plant Med. 68: 5-15.
9. Martins, HM., Martins, ML., Dias, MI., Bernardo, F. (2001). Evaluation of microbiological quality of medicinal plants used in natural infusions. Int J Food Microbiol. 68: 149-153.

10. Elshafie, AE., Al-Rashdi, TA., Al-Bahry, SN., et al. (2002). Fungi and aflatoxins associated with spices in the Sultanate of Oman. Mycopathologia. 155: 155-160.

11. Reif, K., Metzger, W. (1995). Determination of aflatoxins in medicinal herbs and plant extracts. J Chromatogr. 692: 131-136.

12. Pinto, TJA., Kaneko, TM., Ohara, MT. (2000). Controle biológico de qualidade de produtos farmacêuticos. Correlatos e cosméticos. São Paulo: ed. Atheneu. p. 75-95.

13. Santos, PRV., Oliveira, ACX., Tomassini, TCB. (1995). Controle microbiológico de produtos fitoterápicos. Farm Bioquim Universidade de São Paulo. 31: 35-38.

14. Pitt, JI., Hocking, AD. (1997). Fungi and Food Spoilage, 2 edition. Blackie Academic \& Professional, London.

15. Samson, RA., Hoekstna, ES., Frisvad, SC. (2004). Introduction to food airborne fungi. The Netherlands, Baarn. Centralbureau voor Shimmecultures. 
16. Klich, M., Pitt, JI. (1988). Differentiation of Aspergillus flavus from Aspergillus parasiticus and other closely related species. Trans British Mycol Society. 99-108.

17. Lin, MT., Mahajan, JR., Dianese, JC., Takatsu, A. (1976). High Production of Kojic Acid Crystals by Aspergillus parasiticus UNBF A12 in Liquid Medium. Appl Environ Microbiol. 32 (2): 298-299.

18. Soares, LM., Rodriguez-Amaya, DB. (1989). Survey of aflatoxins, ochratoxin A, zearalenone, and sterigmatocystin in some Brazilian foods by using multi-toxin thin-layer chromatographic method. J Assoc Off Anal Chem. 72 (1): 22-6.

19. Dalcero, AC., Magnoli, M., Luna, G., et al. (1998). Mycoflora and naturally occurring mycotoxins in poultry feeds in Argentina. Mycopathologia; 141: 37-43.

20. Gimeno, A. (1980). Improved method for thin layer chromatographic analysis of mycotoxins. J. Assoc of Off. Anal. Chem. 63: 182-186.

21. Scott, PM., Lawrence. (1987). Liquid chromatographic determination and stabilits of the Fusarium Mycotoxin Moniliformin in cereal grains. J Assoc Off Ana. Chem. 70: 850-853.

22. Ministério da Agricultura, Resolução 183 de 21/03/1996.

23. Farmacopéia Brasileira Parte 1. (1988). 4a edição. São Paulo: Editora Atheneu.

24. Corrêa, TBS., Rodrigues, HR., Vargas, EA., et al. (2000). Evaluation of the incidence of mycotoxins in Brazilian maize. 10th International IUPAC Symposium on Mycotoxin and Phycotoxin, São Paulo. p. 20-25.
25. Amorim, SS., Silva, CMG., Pires, RA., et al. (2000). Occurrence of mycotoxins in food and feed in Brazil. 10th International IUPAC Symposium on Mycotoxin and Phycotoxin, São Paulo. p. $20-25$.

26. Abdulkadir, E., Elshafie, AE., Taghreid, AI., et al. (1999). Fungi associated with black tea and tea quality in the Sultanate of Oman. Mycopathologia. 145: 89-93.

27. Tassaneeyakul, W., Razzazi-Fazeli, E., Porasuphatana, S., et al. (2004). Contamination of aflatoxins in herbal medicinal products in Thailand. Mycopathologia. 158: 239-244.

28. Halt, M. (1998). Moulds and mycotoxins in herb tea and medicinal plants. Eur J Epidemiol. 14: 269-274.

29. Food and Agriculture Organization of the United Nations (FAO). (1997). Worldwide regulations for mycotoxins. A Compendium. 64: 7-28.

30. Abarca, ML., Bragulat, MR., Castella, C., et al. (1994). Ochratoxin production by strain of Aspergillus niger var niger. Appl Environ Microbiol. 60 (7): 2650-2652.

31. Dvorackora, I. (1989). Aflatoxins and Human Health. Boca Raton, FL: 1 CRC.

32. Bugno, A., Almodovar, AAB., Pereira, TC., Pinto, TJA., Sabino, M. (2006). Occurrence of toxigenic fungi in herbal drugs. Braz. J. Microbiol. 37, 47-55.

33. Aquino, S., Gonçalez, E., Rossi, MH., Nogueira, JHC., Reis, TA., Correa, B. (2010). Evaluation of fungal burden and aflatoxin presence in packed medicinal plants treated by gamma radiation. J. Food Prot. 73, 932-937. 